\title{
Potential of cellulose paper coated with silver nanoparticles: a benign option for emergency drinking water filter
}

\begin{abstract}
Point-of-use system is an appropriate choice in emergency situation because this system is efficient, easy to use, and highly portable. The present study aimed to determine the optimal parameter values associated with the preparation of cellulose paper coated with silver nanoparticles using response surface methodology via Box-Behnken Design (BBD) for the purpose of finding the appropriate filter material in point-of-use water purification system. In this case, the efficiency of cellulose paper coated with silver nanoparticles was investigated based on the optimal parameter values and tested using different types of water samples (tapwater, rainwater, and surface water) by taking into account the drinking water guidelines and health risks. Finally, the cellulose paper coated with silver nanoparticles was compared with other point-of-use technologies in determining its suitability during emergency situation. Box Behnken Design results showed that quadratic model was capable of describing the effects of parameters (silver nitrate solutions, sodium borohydride; silver nitrate ratio and immersion time) on the responses (E. coli removal efficiency). Moreover, second order polynomial equations, ANOVA, and three-dimensional surface plots were developed to evaluate the effects of each parameter on E. coli removal efficiency. The findings show that optimal parameters are found in silver nitrate concentration of $0.035 \mathrm{M}$ and sodium borohydride: silver nitrate ratio of 5 with insignificant immersion time. The field testing results show that cellulose papers coated with silver nanoparticle is suitable for low-turbidity water as it meets the drinking water guidelines with low health risks. The comparison with other point-of-use technologies indicated that cellulose paper coated with silver nanoparticle is suitable to be used as a filter to supply clean drinking water in emergency situation.
\end{abstract}

Keyword: Drinking water; Emergency; Cellulose paper; Silver nanoparticles; Box-Behnken design; Performance efficacy 